\section{Unrecognised displacement of mandibular molar root into the submandibular space}

\author{
M. A. Nusrath ${ }^{1}$ and R. J. Banks ${ }^{2}$
}

VERIFIABLE CPD PAPER

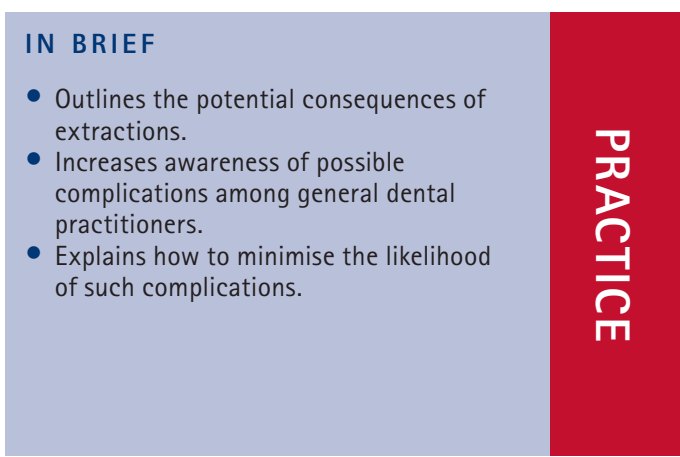

We describe a case of swelling in the right submandibular and sublingual space caused by displacement of a lower second molar root in the submandibular space. This displacement was not recognised at the time of extraction. The techniques used to minimise the risk of accidental displacement of teeth and roots, during extraction are discussed. The importance of recognising this complication and methods of retrieval are highlighted.

\section{INTRODUCTION}

Accidental displacement of teeth or roots can occur during extraction. Lingually inclined teeth or deeply impacted mandibular molar teeth, may have a higher risk of being displaced into the soft tissues. Elevation may also lead to displacement.

Mandibular molar teeth, especially third molars, may be pushed into the sublingual space, submandibular space, pterygomandibular space ${ }^{1}$ and cervical spaces in the neck. Open surgical techniques, application of correct force and placement of lingual retractors may help minimise this particular complication.

We present a case report of a 29-yearold female who was referred via accident and emergency to our department, three months after extraction of a right mandibular second molar with right-sided submandibular and sublingual swelling.

\section{CASE REPORT}

A 29-year-old female was referred by accident and emergency to the maxillofacial unit with right-sided submandibular and sublingual swelling of four days duration.

"Specialist Registrar in Oral \&t Maxillofacial Surgery ${ }^{2}$ Consultant in Oral \& Maxillofacial Surgery, Department of Oral \&t Maxillofacial Surgery, Sunderland Royal Infirmary, Sunderland, SR4 7TP

${ }^{*}$ Correspondence to: Mr Muzzammil Nusrath

Email: manusrath@yahoo.com

\section{Refereed Paper}

Accepted 17 June 2010

DOI: $10.1038 /$ sj.bdj.2010.816

${ }^{\circledR}$ British Dental Journal 2010; 209: 279-280

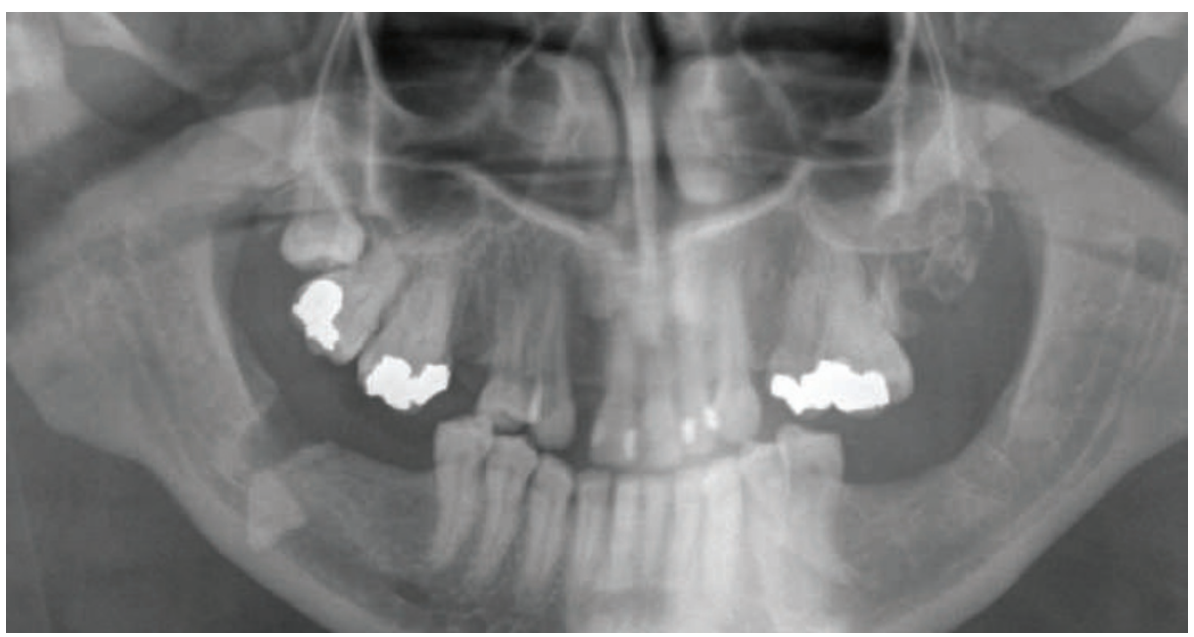

Fig. 1 OPT showing displaced right mandibular molar root in the submandibular space

Three months before the presentation, the patient had undergone extraction of a lower right second molar by the general dental practitioner (GDP). The patient's history suggested that the extraction had proved to be difficult and protracted.

The patient had persistent pain in the right submandibular region for which she subsequently revisited the GDP a week before presentation to accident and emergency. On this occasion, the dental treatment involved extraction of the upper left seven and debridement of the socket of the lower right seven.

Clinical examination revealed trismus; the patient was pyrexial at 37.5 degrees. An orthopantamograph was taken which showed a displaced root fragment in the submandibular space (Fig. 1). The patient was unaware of this and we presume that the dentist also did not notice that part of the distal root of the right second molar had been misplaced.

Under general anaesthesia a $3 \mathrm{~cm}$ crestal incision was placed starting distally from the external oblique ridge, extending onto the crest of the alveolar ridge and ending mesial to the lower right first molar. Lingual subperiosteal dissection was carried out taking care not to damage the lingual nerve. The root remnant was palpated externally in the neck below the submandibular gland. Digital pressure was applied upwards and inwards. Lingual to the socket of the second molar, the index finger of the left hand palpated the mylohyoid muscle, guiding the root remnant into the oral cavity.

The submandibular space was irrigated with normal saline and a corrugated rubber drain placed in situ and sutured. Forty-eight hours later the drain was 
removed when the swelling had subsided. Postoperative lingual and hypoglossal nerve function was normal. Subsequently, the patient was discharged with oral antibiotics and analgesics.

\section{DISCUSSION}

The complication of displacing lower molar teeth/roots is rare. The majority of case reports are for the displacement of lower wisdom teeth. ${ }^{1}$

General dental practitioners extracting lower molar teeth should evaluate the degree of impaction, position of the roots, distal inclination, bone density and if appropriate surgical access should be planned.

Adequate exposure of the surgical site in the form of a muco periosteal flap, proper instrumentation, delivering the appropriate amount and direction of force for elevation of lower molars is very important. If necessary a distal retractor or a finger can be placed lingually to prevent lingual displacement of the crowns or roots. The lingual plate is very thin in the region of the third molar and hence it is easy to perforate the lingual plate; this may lead to displacement of the teeth/roots into the sublingual space, submandibular space and pterygomandibular space.

Upper molars, especially impacted upper third molars, are more prone to be displaced into the infratemporal fossa. Hence placement of a distal retractor to prevent this dislodgement is important. Upper premolar roots and first molar roots may be dislodged into the maxillary sinus.

The mylohyoid muscle divides the sublingual space and the submandibular space. The sublingual space is located superior to the muscle and the submandibular space, inferior to the muscle but superior to the hyoid bone.
There are no posterior fascial borders limiting the sublingual and submandibular spaces. In addition, no fascial border separates these spaces from the inferior parapharyngeal space. Thus, there is free communication between these spaces.

Although some surgeons are of the opinion to delay the removal of such displaced roots and teeth with the argument that the delay favours fibrosis, we do not recommend any delay in removing the fragments, especially if the extraction was due to infectious aetiology. Consider active intervention if the fragment is more than $5 \mathrm{~mm}$. If less than $5 \mathrm{~mm}$ and the fragment is not palpable, then initial management of the case may be conservative. Obtain baseline $\mathrm{X}$ rays, inform the patient about the possibility of infection and arrange follow up in two to three months time.

If the fragment is left for a prolonged period of time there may be a foreign body reaction and also the possibility of infection in the neck spaces with a potential for migration of the fragment into the deeper tissue planes.

Although retrieval of the root in this particular case was carried out with the aid of a panaromic radiograph only, other radiographs like an occlusal, submentovertex and postero-anterior view of the mandible may be helpful in localising the fragment. CT scans should be considered if the fragment is in a deeper neck space. ${ }^{2}$

Intraoperatively the use of image intensifiers may also be very helpful to localise the fragment and theatre staff should be aware of such a possibility, so that appropriate arrangements can be made for the image intensifiers to be made available.

We managed to retrieve this fragment with an intraoral incision only, but sometimes it may be necessary to do a combined intraoral and extraoral approach with a submandibular incision in the neck. ${ }^{3}$

A modified approach for removing fragments displaced lingually is to osteotomise the lingual plate and then approach the fragment, however, in this case we did not have to resort to this. If the fragment is displaced into the pterygomandibular and para pharyngeal space a CT scan is invaluable and in addition tonsillectomy may have to be considered to remove the fragment.

In this particular case the dentist and consequently the patient were unaware that the root had been displaced in the first place and such cases may prove to be a dilemma and a diagnostic challenge when these patients present months later with swelling to accident and emergency. If unrecognised, this could lead to potentially life threatening complications, including deep neck infections, mediastinitis and airway compromise.

When a tooth or root does get displaced it is very important to recognise that such a event has occurred and the patient informed about the complication immediately.

The operator should ensure that the patient's airway is intact. Consider panaromic radiography and suture the wound loosely with dissolvable sutures and immediately refer to the nearest oral and maxillofacial unit as an emergency. If there is going to be a delay for any reason then consider prescribing antibiotics.

1. Ertas U, Selim Yaruz M, Tozoglu S. Accidental third molar displacement into the lateral pharyngeal space. J Oral Maxillofac Surg 2002; 60: 1217.

2. Huang I-Y, Chen C-M, Chang S-W et al. Surgical management of accidentally displaced mandibular third molar into the pterygomandibular space: a case report. Kaohsiung J Med Sci 2007; 23: 370-374.

3. Yeh C-J. A simple retrieval technique for accidentally displaced mandibular third molars. J Oral Maxillofac Surg 2002; 60: 836-837. 